\title{
Oncologist
}

\section{Palliative Care in Advanced Cancer Patients: How and When?}

\author{
Eduardo Bruera, Sriram Yennurajalingam \\ Department of Palliative Care and Rehabilitation Medicine, The University of Texas MD Anderson Cancer \\ Center, Houston, Texas, USA \\ Key Words. Early palliative care • Efficacy of palliative care $•$ Symptom control • Cost of care • \\ Quality care $\cdot$ Supportive care
}

Disclosures: Eduardo Bruera: None; Sriram Yennurajalingam: None.

Section Editor: Russell K. Portenoy: Arsenal Medical Inc., Grupo Ferrer, Xenon (C/A); Ameritox, Archimedes Pharmaceuticals, Boston Scientific, Covidien Mallinckrodt Inc., Endo Pharmaceuticals, Forest Labs, K-Pax Pharmaceuticals, Meda Pharmaceuticals, Medtronics, Otsuka Pharma, ProStrakan, Purdue Pharma, Salix, St. Jude Medical (RF).

Reviewer “A”: Pfizer, Lilly, Bristol-Myers Squibb, KangLaiTe, Alexion, Biovex, DARA, MiCo, NIH, Robert Wood Johnson Foundation, AHRQ (RF); Amgen, Helsinn Therapeutics, Novartis (C/A, RF).

Reviewer "B": None.

(C/A) Consulting/advisory relationship; (RF) Research funding; (E) Employment; (H) Honoraria received; (OI) Ownership interests; (IP) Intellectual property rights/inventor/patent holder; (SAB) Scientific advisory board

\section{LEARNING OBJECTIVES}

After completing this course, the reader will be able to:

1. Discuss the impact of early access to palliative care service on symptom control, quality of life, and cost of care.

2. Formulate strategies to improve early palliative care access through education and research.

\section{GME} This article is available for continuing medical education credit at CME. TheOncologist.com.

\section{Abstract}

Cancer patients develop severe physical and psychological symptoms as a result of their disease and treatment. Their families commonly suffer great emotional distress as a result of caregiving. Early palliative care access can improve symptom control and quality of life and reduce the cost of care. Preliminary results show that early palliative care access can also extend survival. Unfortunately, only a minority of cancer centers in the U.S. have
\end{abstract}

the two most important resources for palliative care delivery: outpatient palliative care centers and inpatient palliative care units. In this article, we use a case presentation to discuss the impact of early palliative care access in light of the currently available evidence, and we recommend ways to improve early access to palliative care through education and research. The Oncologist 2012;17: 267-273

\section{Patient Story}

Mr. S was a 52-year-old man seen as a new outpatient at our cancer center. He had recently been diagnosed with stage III non-small cell carcinoma of the lung with a $3-\mathrm{cm}$ nodule in the right lower lobe and hilar and mediastinal adenopathies. After initial evaluation, the patient was started on a combined regimen of radiation therapy, chemotherapy, and targeted therapy.
At the time of the initial evaluation, the thoracic medical oncologist referred the patient to the supportive care center, where a full assessment was conducted by a palliative medicine team. During the initial assessment by the supportive care team, the patient was found to have chest pain, nausea, moderate dyspnea, and severe fatigue. He was prescribed slow-release morphine, senna, and metoclopramide to treat these symptoms. In addition, the pa-

Correspondence: Eduardo Bruera, M.D., Palliative Care \& Rehabilitation Medicine, Unit 1414, The University of Texas MD Anderson Cancer Center, 1515 Holcombe Boulevard, Houston, Texas 77030, USA. Telephone: 713-792-6084; Fax: 713-792-6092; e-mail: ebruera@mdanderson.org Received June 29, 2011; accepted for publication October 12, 2011; first published online in The Oncologist Express on January 17, 2012. CAlphaMed Press 1083-7159/2012/\$40.00/0 http://dx.doi.org/10.1634/theoncologist.2011-0219 
tient underwent expressive supportive counseling with one of the palliative trained counselors and was prescribed increased physical activity as well as exposure to natural light for the management of fatigue and insomnia.

Over the next 2 months, the patient was seen on the same day by the medical oncologist at the thoracic center and the palliative medicine specialist at the supportive care center. Because of progressive disease in the mediastinum and chest wall, the patient started second-line therapy. At the supportive care center, the patient was found to have increased fatigue and sedation. The patient was prescribed methylphenidate, and the patient's wife and child received counseling.

One month later, the patient developed back pain caused by bony metastases to the lumbar spine and was started on palliative radiation therapy. In addition, because the patient continued to experience sedation and had begun to experience myoclonus, the opioid was switched to methadone, the laxative dose was increased, and the patient was provided with a disabled parking placard, occupational therapy, and a home safety evaluation.

Two months later, because his disease had progressed, the patient was referred by the thoracic oncologist to our institution's Center for Targeted Therapy for consideration of experimental treatment in a phase I clinical trial. On the same day as his first phase I pretreatment evaluation, the patient was seen at the supportive care center. The doses of his opioid and antiemetic were adjusted, a corticosteroid was added for the management of fatigue and pain, and the methylphenidate dose was adjusted.

After two courses of targeted therapy in the trial, the patient was found to have progressive disease. At that point, because of deterioration in his performance status, the patient and his family chose to discontinue treatment. On the same day, the patient was assessed at the supportive care center and was found to have delirium with mild psychomotor agitation and hallucinations as well as increased pain intensity. The primary medical oncologist and the palliative care physician agreed that the patient would be admitted to the palliative care unit under the care of a palliative care physician. During the patient's 5-day stay in the unit, he underwent opioid rotation and parenteral hydration, he was given haloperidol for agitation and antibiotics for pneumonia, and a family conference was conducted. The patient's delirium resolved, and he was discharged home with hospice care; his symptoms remained well controlled until his death 3 weeks later.

Mr. S received medical oncology and palliative care simultaneously from almost the moment of diagnosis to death. In a recent randomized controlled study of 151 patients with advanced lung cancer, Temel et al. [1-3] observed that patients allocated to palliative care referral with standard care had a better quality of life (as shown by their Functional Assessment of Cancer TherapyLung scores), less depression (as shown by their Hospital Anxiety and Depression Scale scores), and a longer median survival time (11.6 months versus 8.9 months) than patients receiving just standard care [1]. Palliative care has also been found to improve symptom control $[2,4,5]$ and reduce the cost of care [6-10].
A

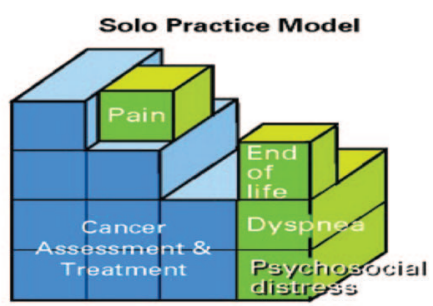

B

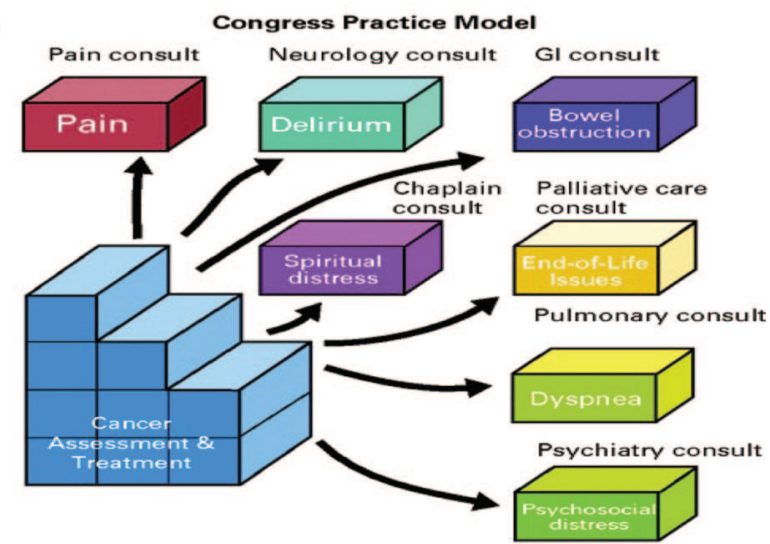

C

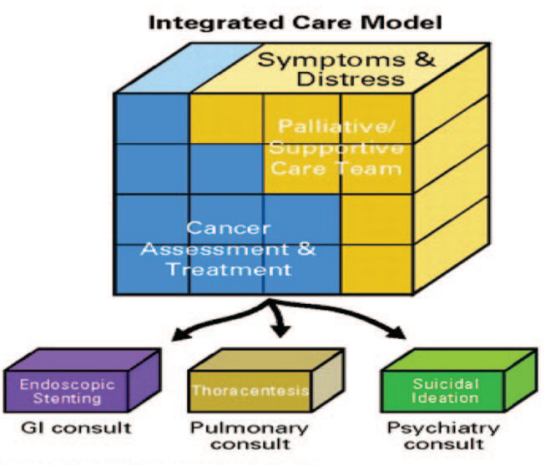

Figure 1. Conceptual model for integration of palliative/supportive care in oncology. (A): Solo practice model. (B): Congress practice model. (C): Integrated care model.

Abbreviation: GI, gastrointestinal.

Reprinted with permission. (C) 2008 American Society of Clinical Oncology. All rights reserved. Bruera E, Hui D. J Clin Oncol 28, 2010:4013-4017.

However, very few patients in cancer centers and acute care facilities [11] receive the type of care $\mathrm{Mr}$. S did.

In the following paragraphs, we discuss the impact of early palliative care access and recommend ways to improve early access through education and research.

\section{Models of Palliative Care Delivery}

Palliative care is provided by a team of health care professionals that can include a primary oncologist, palliative care specialist, registered nurse trained in palliative care, chaplain, social worker, pharmacist, counselor, occupational therapist, and physiotherapist.

The role of the palliative care team is to assess and manage patients' and families' care needs in the physical, psychological, social, spiritual, and information domains. This care includes the 
pharmacological management of symptoms, education and counseling for psychosocial and spiritual issues, help with advance directives, and family support and education.

The oncologist's care of cancer patients requires complex multidisciplinary interventions. These interventions include diagnosis of the disease and determination of its stage, development of a complex treatment plan in coordination with other team members (e.g., radiation and surgical oncologists), and management of cancer treatment and its complications.

These are time-consuming tasks that make it increasingly difficult for a busy oncologist to address multiple supportive/ palliative care needs in the same visit. The lack of time is becoming an even larger concern as the body of knowledge and available interventions become more complex in oncology and palliative/supportive care.

Figure 1 illustrates three models for the integration of oncology and palliative/supportive care [12]. In the solo practice model (Fig. 1A), "the oncologist takes care of all the disease assessments, management as well as all supportive/palliative care needs. This model is commonly required in community practices where supportive/palliative care consultants are unavailable. The key advantage is that the patient receives all aspects of care from the same oncologist. The disadvantage includes time constraints, limited palliative training and a risk of burnout of the oncologist as a result of the need to assume all aspects of care" [12]. To deliver high-quality supportive/palliative care under this model, the solo practitioner needs to receive extensive training.

In the congress approach model (Fig. 1B), the oncologist refers patients to multiple consultants, such as a pain specialist for pain and a psychiatrist for emotional distress. The congress model has limitations, which include fragmented communication among the health care providers themselves and between physicians and patients and their families; this model can be time-consuming and exhausting for patients and expensive for patients and the health care system. The main risks of the congress model include potential polypharmacy, and in some instances, conflicting care plans, especially when there is limited communication among consulting specialists.

The integrated-care model between the primary oncologist and a supportive/palliative care team (Fig. 1C) allows the oncologist to focus on all aspects of cancer management while the supportive/palliative care team addresses physical symptoms and psychosocial concerns. This model includes consultation of other specialists for specific reasons, such as pulmonologists for interventional bronchoscopy aimed at relieving bronchial obstruction. However, because most problems can be managed by the supportive/palliative care team, there are fewer visits and lower costs. Taking advantage of these established teams allows the oncologist to focus on the complex issues associated with the management of cancer.

\section{Benefits of Palliative Care}

\section{Symptom Control}

Studies by our group and others [13-18] have shown improved symptom control in patients with advanced cancer as a result of a palliative care consultation. In a recent study, our group re- viewed 406 new consultations seen at our outpatient palliative care clinic [4]. We found that, at the first follow-up visit after the consultation, patients achieved significant improvements in most cancer-related symptoms. Studies by Follwell et al. [17] and Rabow et al. [16] also showed significant improvements in the symptoms of patients receiving palliative care in the outpatient setting. Similar improvements in physical and psychological symptoms and other patient-related quality-ofcare outcomes were found when palliative care was delivered by inpatient palliative care units [19], mobile team consultations [20,21], and intensive care unit palliative care consultations [22]. In a recent study by Casarett et al. [23], patients admitted to palliative care units achieved better symptom control than patients seen by palliative care teams at regular sites of care (i.e., medical/surgical wards, intensive care units, and nursing homes). These results are very impressive because patients admitted to palliative care units have more severe symptom distress than patients in other inpatient settings [4, 5, 19, $21,22,24]$.

There are numerous reasons why palliative care is more capable of improving symptoms than standard oncology care. Palliative care teams conduct systematic symptom evaluations, using standard tools such as the Edmonton Symptom Assessment System [25, 26], and document symptom distress on a regular basis. In addition, palliative care teams provide patients with regular assessments of emotional distress, screenings for delirium, and screenings for risk factors for the misuse of opioids using tools such as The Cut Down, Annoyed, Guilty, and Eye Opener (CAGE) questionnaire [27]. Palliative care inpatients and outpatients have access to an interdisciplinary team capable of rapidly managing physical, emotional, functional, social/family, and spiritual problems. Because the vast majority of patients starting treatment with medical oncologists have a number of these problems, it is likely that most of these patients would benefit from early palliative care consultations.

\section{Lower Cost}

A disproportionate amount of resources is spent at the end of life: $30 \%$ of Medicare expenditures are attributable to the $5 \%$ of beneficiaries who die each year [28] and about one-third of the expenditures in the last year of life are spent in the last month of life [29]. Several studies by our team and others [6, 8, 9, 30-34] found that patients with advanced disease, including those with cancer, who received palliative care consultations had lower medical costs at their end of life. These investigators also found that higher costs were associated with a worse endof-life experience. A recent study by Morrison et al. [6] found that palliative care consultation teams can lower hospital costs for Medicaid patients with advanced illnesses. Based on data from four diverse urban New York hospitals, the authors concluded that the state of New York could save an estimated $\$ 84-\$ 252$ million annually if every hospital with $\geq 150$ beds had a fully operational palliative care consultation team. However, the association between health care expenditures and outpatient palliative care consultation at the end of life in patients with advanced cancer has not been fully examined. 
There are a number of possible reasons why a palliative care consultation is capable of reducing the cost of care. Among the most important of these are that palliative care might reduce intensive care unit deaths, reduce the number of days patients spend in acute inpatient care [24], and help avoid the use of unnecessary and excessive procedures [1]. The simultaneous discussion of potential end-of life care and cancer treatment is likely to allow patients and their families to better adjust to events such as progressive disease and the need to discontinue therapy.

\section{Survival}

Studies by Temel et al. [1] and Bakitas et al. [35] showed preliminary evidence for a survival benefit in patients who received palliative care. This benefit may be a result of multiple factors. First, better symptom control and physical and psychosocial function could lead to a better ability to adhere to cancer treatment regimens. Second, physical (i.e., pain, dyspnea, fatigue) and emotional (i.e., anxiety, depression) distress have been linked to greater mortality related to cancer [36] and other conditions [37-39]. Bakitas et al. [35], Temel et al. [1, 35], and our team $[4,21,22]$ found that palliative care access resulted in less depression and anxiety. Finally, effective transition to end-of-life care might prevent patients from receiving potentially harmful interventions $[1,40]$. However, further, larger studies with overall survival as a primary outcome are needed.

\section{Role of Palliative Care in Providing QUALITY CARE}

In an atmosphere of renewed focus on health care reform, there has been more emphasis on provision of quality care. The Institute of Medicine's Crossing the Quality Chasm report [41, 42] focused on improving the delivery of quality care by the adoption of six specific aims (that care be safe, beneficial, timely, efficient, patient centered, and equitable). These six aims are the basis of the quality assessment and performance improvement recommendations of the National Consensus Panel [43], National Quality Forum [42], Joint Commission [44], and American Society of Clinical Oncology [45-47]. Some of the outcome measures for quality care include: the percentage of patients who die without access to hospice care or receive hospice care $\leq 3$ days before death or chemotherapy $\leq 14$ days before death, intensive care unit admissions $<30$ days before death, emergency room visits near the end of life, and the percentage of patients who experience poor symptom control. Palliative care is perceived as a highly effective service for improving many of these metrics for quality cancer care $[1,9,48]$.

\section{AChieving Integrated Oncology-Palliative Care Practice}

Studies have shown that patients with advanced lung cancer (stage III or stage IV) receiving potentially curative therapy, such as the patient described in the case report, are likely to have severe physical and psychological symptoms $[49,50]$. These patients and their families also require extensive education and counseling regarding the spiritual, financial, and family distress imposed by the diagnosis of a devastating disease with a typically poor prognosis. Hence, early referral to palliative/supportive care can be extremely beneficial.

Early referral to palliative care has been shown to facilitate timely diagnosis and treatment of symptoms, longitudinal psychosocial support and counseling, and a gradual transition of care [2, 4, 13-18, 51]. Palliative care referrals have also been shown to reduce end-of-life care costs [6-9, 29-33]. However, traditional palliative care practice is predominantly based on the delivery of care by inpatient consultation teams and/or in palliative care units. Currently, only $66 \%$ of comprehensive cancer centers and $29 \%$ of regional cancer centers in the U.S. have outpatient services capable of delivering early palliative care [11]. Even in centers where such outpatient programs are available, late referrals, reflecting a sequential practice by the medical oncologist, have been a consistent problem [52, 53]. This problem is particularly important in patients with hematological malignancies [53]. Research by our group [19] and others [23] has shown that inpatient palliative care units are effective in rapidly relieving symptoms that seemed intractable. However, only $26 \%$ of comprehensive cancer centers and $20 \%$ of regional cancer centers in the U.S. report having dedicated inpatient palliative care beds [11].

Expected changes in reimbursement for cancer care, whereby payments are made according to pay-for-performance initiatives that are based on quality cancer care metrics, will provide a strong incentive for institutions to develop the structures and processes that allow early palliative care access in most medium- and large-sized cancer centers. In smaller institutions, there will be a need for a combined approach that will supplement increased direct supportive/palliative care delivery by medical oncologists with initiatives such as bridging palliative care programs [54], the use of technologies such as video conferencing, traveling palliative care specialists, and even designated palliative care experts within oncology teams.

Early access to palliative care can be achieved by intense collaboration between medical oncologists and palliative care teams, which must be based on mutual trust. Health care professionals frequently have concerns that early referral to supportive/palliative care could have negative effects on patients' hope (e.g., a feeling of "pulling the plug") [55]. As a result, our team has developed an educational approach that can be useful to health care professionals and to patients and their families; this approach is based on a concept called "goals of car."

The approach's adoption of an analogy to the purchase of a car is useful because the majority of patients and their families are familiar with buying cars. Figure 2 shows the expected goals when purchasing a car. Without changing the primary goals of traveling and getting to work, car owners do take precautions in case things go bad, such as buying insurance, wearing seatbelts, and having air bags. An attitude of expecting the primary goal to be reached successfully without taking any minimal precautions would be seen as extremely unrealistic. Similarly, although patients can maintain goals such as cure, life prolongation, cancer treatment, and participation in clinical trials, the referral to supportive and palliative care allows patients to receive a number of comfort measures, such as treatment for physical and emotional distress, as well as safety 

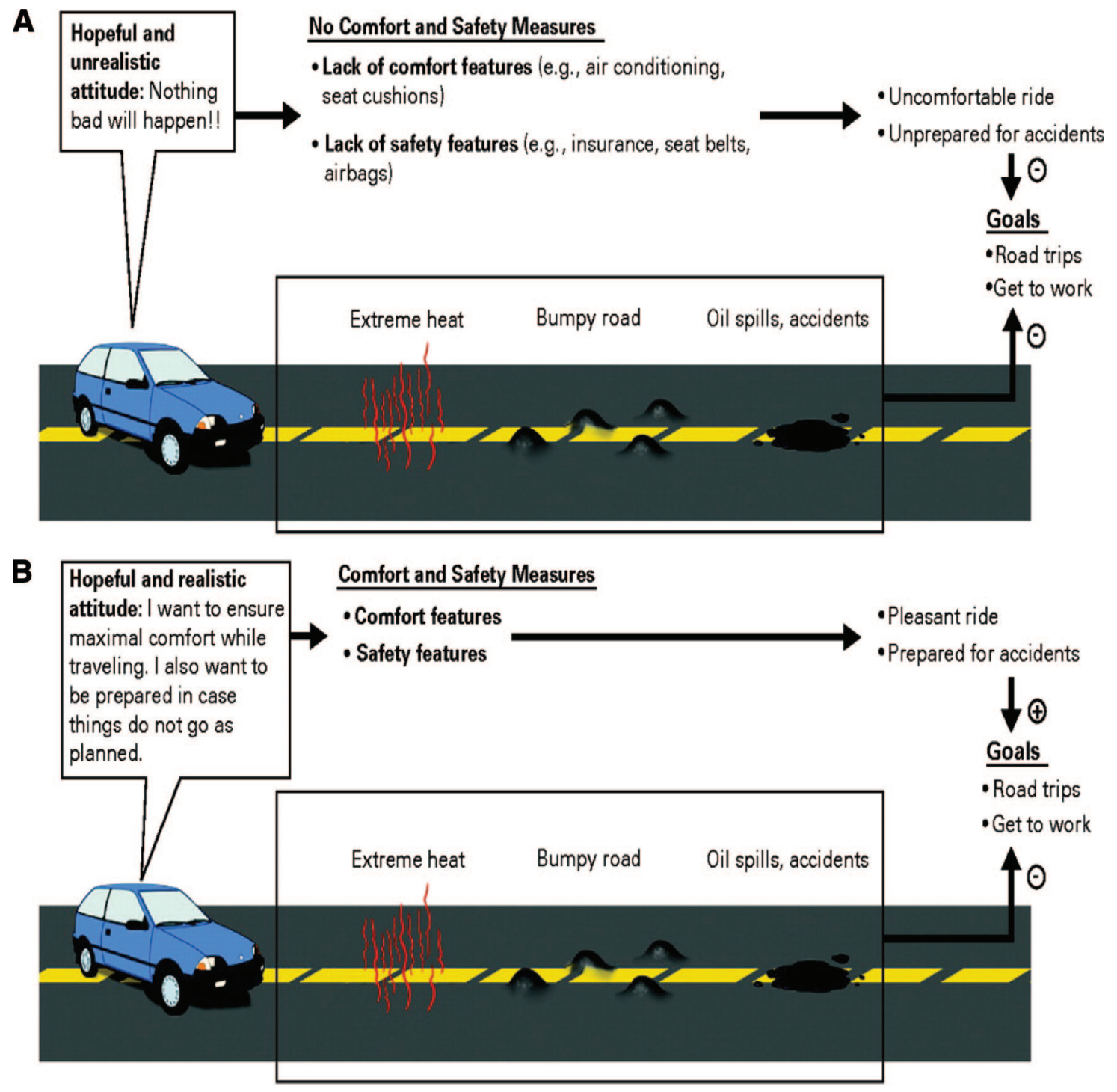

Q 2010 The University of Texas M.D. Anderson Cancer Center.

Figure 2. Goals for the use of a car are analogous to goals of care. (A): No Comfort and safety measures. (B): Comfort and safety measures.

Reprinted with permission. (C) 2008 American Society of Clinical Oncology. All rights reserved. Bruera E, Hui D. J Clin Oncol 28, 2010:4013-4017.

measures, such as advanced care, planning for living arrangements, transportation, bedroom and bathroom aids, etc.

Fadul et al. [55], in a survey of 100 medical oncologists and 100 midlevel providers at our institution, found a greater likelihood of patient referral to a service named "supportive care" than to one named "palliative care." We used these findings to improve early access to palliative care by changing the name of our outpatient and inpatient consultation service from "palliative care" to "supportive care" in November 2007. In a recent retrospective study of 4,701 consecutive patients [48], we found a $41 \%$ increase in the number of referrals after the name change. In the outpatient setting, the time duration between supportive/palliative care consultation and death or last follow-up was significantly longer (6.2 months versus 4.7 months; hazard ratio, $1.21 ; p<.001)$. The per- centage of patients referred with early-stage cancer increased from $5 \%$ to $14 \%$.

\section{The Nature of Palliative Care Intervention}

Previous randomized controlled trials and systematic reviews $[56,57]$ found that simple, low-cost palliative care interventions were generally unable to significantly influence symptoms, place of death, and other clinical variables in a number of patient populations. More recent studies conducted with comprehensive interdisciplinary palliative care teams led by palliative medicine specialists have demonstrated beneficial effects on symptom distress $[4,5,23]$, decreased costs of care, and fewer intensive care unit admissions [19, 24]; preliminary results suggest that there might even be a longer median survival 
duration for patients receiving early palliative care [1]. However, there are no published data that characterize the most useful components of a palliative care intervention, namely, the palliative care specialist, nurse, social worker, chaplain, and counselor.

Hence, there is a great need for future clinical trials to better define the ideal timing and most useful components of palliative care consultation and to determine the effectiveness of palliative care consultation in improving quality care outcomes for different cancer types and clinical settings.

\section{Conclusions}

Early palliative care access can improve symptom control and patient quality of life and reduce the cost of care. Unfortunately, a minority of cancer centers in the U.S. have the two most important resources for palliative care delivery: outpatient palliative care centers and inpatient palliative care units. Combined work by medical oncology and supportive/palliative care teams has the potential to greatly improve patient care.

\section{ACKNOWLEDGMENT}

This research is supported in part by the National Institutes of Health through MD Anderson's Cancer Center Support Grant, CA016672.

\section{Author Contributions}

Conception/Design: Eduardo Bruera, Sriram Yennurajalingam

Provision of study material or patients: Eduardo Bruera, Sriram Yennurajalingam

Collection and/or assembly of data: Eduardo Bruera, Sriram Yennurajalingam

Data analysis and interpretation: Eduardo Bruera, Sriram Yennurajalingam

Manuscript writing: Eduardo Bruera, Sriram Yennurajalingam

Final approval of manuscript: Eduardo Bruera, Sriram Yennurajalingam

\section{REFERENCES}

1. Temel JS, Greer JA, Muzikansky A et al. Early palliative care for patients with metastatic nonsmall cell lung cancer. N Engl J Med 2010;363: 733-742.

2. Jacobsen J, Jackson V, Dahlin C et al. Components of early outpatient palliative care consultation in patients with metastatic nonsmall cell lung cancer. J Palliat Med 2011;14:459-464.

3. Arafah BM. Hypothalamic pituitary adrenal function during critical illness: Limitations of current assessment methods. J Clin Endocrinol Metab 2006;91:3725-3745.

4. Yennurajalingam S, Urbauer DL, Casper KL et al. Impact of a palliative care consultation team on cancer-related symptoms in advanced cancer patients referred to an outpatient supportive care clinic. J Pain Symptom Manag 2011;41:49-56.

5. Bruera E, Michaud M, Vigano A et al. Multidisciplinary symptom control clinic in a cancer center: A retrospective study. Support Care Cancer 2001;9:162-168

6. Morrison RS, Dietrich J, Ladwig S et al. Palliative care consultation teams cut hospital costs for Medicaid beneficiaries. Health Aff (Millwood) 2011;30:454-463.

7. Morrison RS, Penrod JD, Cassel JB et al. Cost savings associated with US hospital palliative care consultation programs. Arch Intern Med 2008;168: 1783-1790.

8. Penrod JD, Deb P, Luhrs C et al. Cost and utilization outcomes of patients receiving hospitalbased palliative care consultation. J Palliat Med 2006;9:855-860.

9. Smith TJ, Coyne P, Cassel B et al. A highvolume specialist palliative care unit and team may reduce in-hospital end-of-life care costs. J Palliat Med 2003;6:699-705.

10. Bruera E, Neumann CM, Gagnon B et al. The impact of a regional palliative care program on the cost of palliative care delivery. J Palliat Med 2000; 3:181-186.

11. Hui D, Elsayem A, De La Cruz M et al. Availability and integration of palliative care at US cancer centers. JAMA 2010;303:1054-1061.

12. Bruera E, Hui D. Integrating supportive and palliative care in the trajectory of cancer: Establishing goals and models of care. J Clin Oncol 2010;28: 4013-4017.

13. Higginson IJ, Evans CJ. What is the evidence that palliative care teams improve outcomes for cancer patients and their families? Cancer J 2010 16:423-435.

14. Lorenz KA, Lynn J, Dy SM et al. Evidence for improving palliative care at the end of life: A systematic review. Ann Intern Med 2008;148:147159.

15. Strömgren AS, Groenvold M, Petersen MA et al. Pain characteristics and treatment outcome for advanced cancer patients during the first week of specialized palliative care. J Pain Symptom Manag 2004;27:104-113.

16. Rabow MW, Dibble SL, Pantilat SZ et al. The comprehensive care team: A controlled trial of outpatient palliative medicine consultation. Arch Intern Med 2004;164:83-91.

17. Follwell M, Burman D, Le LW et al. Phase II study of an outpatient palliative care intervention in patients with metastatic cancer. J Clin Oncol 2009 27:206-213.

18. Hanks GW, Robbins M, Sharp D et al. The imPaCT study: A randomised controlled trial to evaluate a hospital palliative care team. Br J Cancer 2002;87:733-739.

19. Elsayem A, Swint K, Fisch MJ et al. Palliative care inpatient service in a comprehensive cancer center: Clinical and financial outcomes. J Clin Oncol 2004;22:2008-2014.

20. Braiteh F, El Osta B, Palmer JL et al. Characteristics, findings, and outcomes of palliative care inpatient consultations at a comprehensive cancer center. J Palliat Med 2007;10:948-955.

21. Yennurajalingam $S$, Zhang T, Bruera E. The impact of the palliative care mobile team on symptom assessment and medication profiles in patients admitted to a comprehensive cancer center. Support Care Cancer 2007;15:471-475.

22. Delgado-Guay MO, Parsons HA, Li Z et al. Symptom distress, interventions, and outcomes of intensive care unit cancer patients referred to a palliative care consult team. Cancer 2009;115:437445.

23. Casarett D, Johnson M, Smith D et al. The op- timal delivery of palliative care: A national comparison of the outcomes of consultation teams vs inpatient units. Arch Intern Med 2011;171:649655.

24. Bruera E, Neumann CM, Gagnon B et al. Edmonton regional palliative care program: Impact on patterns of terminal cancer care. CMAJ 1999;161: 290-293.

25. Dudgeon D, King S, Howell D et al. Cancer Care Ontario's experience with implementation of routine physical and psychological symptom distress screening. Psychooncology 2011 Feb 8 doi: 10.1002/pon.1918 [Epub ahead of print].

26. Barbera L, Seow H, Howell D et al. Symptom burden and performance status in a populationbased cohort of ambulatory cancer patients. Cancer 2010;116:5767-5776.

27. O'Leary NS, Lawlor PG. Multidimensional assessment: Pain and palliative care. In: Bruera E, Portenoy RK, editors. Cancer Pain, Assessment and Management, Second Edition. Cambridge, U.S.: Cambridge University Press, 2010:105-129.

28. Barnato AE, McClellan MB, Kagay CR et al. Trends in inpatient treatment intensity among Medicare beneficiaries at the end of life. Health Serv Res 2004;39:363-376.

29. Emanuel EJ, Ash A, Yu W et al. Managed care, hospice use, site of death, and medical expenditures in the last year of life. Arch Intern Med 2002;162:1722-1728.

30. Zhang B, Wright AA, Huskamp HA et al. Health care costs in the last week of life: Associations with end-of-life conversations. Arch Intern Med 2009;169:480-488.

31. Gade G, Venohr I, Conner D et al. Impact of an inpatient palliative care team: A randomized controlled trial. J Palliat Med 2008;11:180-190.

32. Penrod JD, Deb P, Dellenbaugh C et al. Hospital-based palliative care consultation: Effects on hospital cost. J Palliat Med 2010;13:973-979.

33. Brumley R, Enguidanos S, Jamison P et al. Increased satisfaction with care and lower costs: Results of a randomized trial of in-home palliative care. J Am Geriatr Soc 2007;55:993-1000.

34. White KR, Stover KG, Cassel JB et al. Nonclinical outcomes of hospital-based palliative care. J Healthc Manag 2006;51:260-274. 
35. Bakitas M, Lyons KD, Hegel MT et al. Effects of a palliative care intervention on clinical outcomes in patients with advanced cancer. JAMA 2009;302:741-749.

36. Pinquart M, Duberstein PR. Depression and cancer mortality: A meta-analysis. Psychol Med 2010;40:1797-1810.

37. Faller H, Bl̈zebruck H, Drings P et al. Coping, distress, and survival among patients with lung cancer. Arch Gen Psychiatry 1999;56:756-762.

38. Barefoot JC, Schroll M. Symptoms of depression, acute myocardial infarction, and total mortality in a community sample. Circulation 1996;93: 1976-1980.

39. Denollet J, Schiffer AA, Spek V. A general propensity to psychological distress affects cardiovascular outcomes: Evidence from research on the type D (distressed) personality profile. Circ Cardiovasc Qual Outcomes 2010;3:546-557.

40. Mitchell SL, Teno JM, Kiely DK et al. The clinical course of advanced dementia. N Engl J Med 2009;361:1529-1538.

41. Institute of Medicine. Crossing the Quality Chasm: A New Health System for the 21st Century. Washington, D.C., National Academy Press, 1-337.

42. National Quality Forum. A National Framework and Preferred Practices for Palliative and Hospice Care Quality: A Consensus Report. Pittsburg, PA. National Quality Forum, 1-71.

43. National Consensus Project for Quality Palliative Care (NCP). Available at http://
www.qualityforum.org/Publications/2006/12/A_ National_Framework_and_Preferred_Practices_for_ Palliative_and_Hospice_Care_Quality.aspx, accessed November 10, 2011.

44. Advanced Certification for Palliative Care Programs. Available at http://www.jointcommission.org/ certification/palliative_care.aspx, accessed November 10, 2011.

45. ASCO-ESMO consensus statement on quality cancer care. J Clin Oncol 2006;24:3498-3499.

46. Ferris FD, Bruera E, Cherny N et al. Palliative cancer care a decade later: Accomplishments, the need, next steps-from the American Society of Clinical Oncology. J Clin Oncol 2009;27:3052-3058.

47. American Society of Clinical Oncology Quality Oncology Practice Initiative (ASCO QOPI). ASCO QOPI Summary of Measures, Fall 2009. Available at http://qopi.asco.org/Documents/ QOPIFall2009MeasuresSummary8-18-09.pdf, accessed September 1, 2011.

48. Dalal S, Palla S, Hui D et al. Association between a name change from palliative to supportive care and the timing of patient referrals at a comprehensive cancer center. The Oncologist 2011;16: 105-111.

49. Wang XS, Fairclough DL, Liao Z et al. Longitudinal study of the relationship between chemoradiation therapy for non-small-cell lung cancer and patient symptoms. J Clin Oncol 2006;24:4485-4491.

50. Wang XS, Shi Q, Williams LA et al. Inflammatory cytokines are associated with the development of symptom burden in patients with
NSCLC undergoing concurrent chemoradiation therapy. Brain Behav Immun 2010;24:968-974.

51. Temel JS, Greer JA, Admane S et al. Longitudinal perceptions of prognosis and goals of therapy in patients with metastatic non-small-cell lung cancer: Results of a randomized study of early palliative care. J Clin Oncol 2011;29: 2319-2326.

52. Morita T, Akechi T, Ikenaga $\mathrm{M}$ et al. Late referrals to specialized palliative care service in Japan. J Clin Oncol 2005;23:2637-2644.

53. Osta BE, Palmer JL, Paraskevopoulos T et al. Interval between first palliative care consult and death in patients diagnosed with advanced cancer at a comprehensive cancer center. J Palliat Med 2008;11:51-57.

54. Casarett D, Abrahm JL. Patients with cancer referred to hospice versus a bridge program: Patient characteristics, needs for care, and survival. J Clin Oncol 2001;19:2057-2063.

55. Fadul N, Elsayem A, Palmer JL et al. Supportive versus palliative care: What's in a name? A survey of medical oncologists and midlevel providers at a comprehensive cancer center. Cancer 2009; 115:2013-2021.

56. El-Jawahri A, Greer JA, Temel JS. Does palliative care improve outcomes for patients with incurable illness? A review of the evidence. J Support Oncol 2011;9:87-94.

57. Zimmermann C, Riechelmann R, Krzyzanowska $\mathrm{M}$ et al. Effectiveness of specialized palliative care: A systematic review. JAMA 2008;299: 1698-1709. 